\title{
Optimal allocation of DG unit for the radial distribution system using genetic algorithm
}

\author{
T. Monish kumar ${ }^{1}$, Dr.V. Ganesh ${ }^{2}$ \\ PG scholar, Dept. of EEE, JNTUA college of Engineering, Pulivendula, Kadapa(dt) A.P, India ${ }^{1}$ \\ Professor, Dept. of EEE, JNTUA college of Engineering, Pulivendula, Kadapa(dt) A.P, India ${ }^{2}$
}

\begin{abstract}
This paper proposes a Genetic Algorithm (GA) based technique for the optimal allocation of Distributed Generation (DG) units in the power systems. In this paper the main aim is to decide optimal number size and location of DG units for voltage profile improvement and power loss reduction in distribution network. Wind based type of DG is considered and distributed load flow is used to calculate exact power loss. Load flow algorithm is combined appropriately with GA till access to acceptable results of this operation. The suggested method is programmed under MATLAB software. The effectiveness of the proposed methodology was tested on Standard IEEE 37 bus system.
\end{abstract}

Keywords:Distributed Generation (DG), Genetic Algorithm (GA),Probability Density Function(PDF), Local Distributed Company(LDC), Radial Distribution system(RDS), Net Present Value(NPV), Risk Factor(RF).

\section{INTRODUCTION}

Environmental and economic aspects leads to change in and sizing is of great importance. The installation of DG the power generation system, with the challenges currently units at non-optimal places can result in an increase in faced by the power generation and the restructure of the system losses, implying in an increase in costs and, energy systems makes the Distribution Generation(DG) as therefore, having an effect opposite to the desired. For that a better option. Renewable energy sources are the only reason, the use of an optimization method capable of resources which are neither exhaustible nor create any indicating the best solution for a given distribution damage to the environment. Improper allocation of DG network can be very useful for the system planning units in the distribution networks lead to harmful effects. engineer. The selection of the best places for installation Effective care and plan should be taken in allocating these and the preferable size of the DG units in large distribution DG units to maximize the benefits satisfying the system systems is a complex combinatorial optimization problem. constrains.

A distribution system provides a final link between high voltage transmission systems and consumer services. The power loss is significantly high in distribution systems because of lower voltages and higher currents, when compared to that in high voltage transmission systems. Studies have indicated that as much as $13 \%$ of total power generated is consumed as $I^{2} R$ losses. Reactive currents account for a portion of these losses. Reduction of total loss in distribution systems is very essential to improve the overall efficiency of power delivery. The pressure of improving the overall efficiency of power delivery has forced the power utilities to reduce the loss, especially at distribution level. The alternative after considering the above factor is to introduce distributed and dispersed generation which can be conveniently located closer to load centres. The main idea behind the distributed Generation (DG) is that generation in small scale and can be easily placed closer to the point of consumption.

The planning of the electric system with the presence of DG requires several factors to be taken into considerations, such as: the best technology to be used, the number and the capacity of the units, the best location, the type of network connection etc. The impact of DG in system operating characteristics, such as electric losses, voltage profile, stability and reliability needs to be appropriately evaluated. The problem of DG allocation Genetic algorithms offer a new and powerful approach to these optimization problems made possible by the increasing availability of high performance computers at relatively low costs. These algorithms have recently found extensive applications in solving global optimization searching problems when the closed-form optimization technique cannot be applied. Genetic algorithms (GAs) are parallel and global search techniques that emulate natural genetic operators.

\section{DISTRIBUTED GENERATIONS}

A general definition was then suggested in which are now widely accepted as follows: "Distributed Generation is an electric power source connected directly to the distribution network or on the customer site of the meter". The definitions of DG do not define the technologies, as the technologies that can be used vary widely. However, a categorization of different technology groups of DG seems possible, such as, non-renewable DG and renewable DG. From distribution system planning point of view, DG is a feasible alternative for new capacity especially in the competitive electricity market environment and has immense benefit such as : Short lead time and low investment risk since it is built in modules, Small-capacity modules that can track load variation more closely, Small physical size that can be installed at load centre and does not need government approval or search for utility territory and land availability, Existence of a vast range of DG technologies. 
For these reasons, the first signs of a possible crossover have been proposed in the literature. Single technological change are beginning to arise on the point crossover has been used in this paper. international scene, which could involve in the future the presence of a consistently generation produced with small and medium size plants directly connected to the distribution network (LV and MV) and characterized by good efficiencies and low emissions. This will create new problems and probably the need of new tools and managing these systems

\section{GENETIC ALGORITHM}

GA is general purpose optimization algorithm based on the mechanics of natural selection and genetics. They operate on string structures (chromosomes), typically a concatenated list of binary digits representing a coding of the control parameters (phenotype) of a given problem. Chromosomes themselves are composed of genes. GA is an attractive alternative to other optimization methods because of their robustness. There are four major differences between GA and conventional optimization algorithms.

i). GA operates on the encoded string of the problem parameters rather than the actual parameters of the problem.

ii). GA uses a population of points rather than a single point in their search.

iii). GA does not require any prior knowledge, space limitations, or special properties of the function to be optimized, such as smoothness, convexity and existence of derivatives. They only require the evaluation of the so called Fitness Function (FF) to assign a quality value to every solution produced.

iv). GA use probabilistic transition rules, not deterministic rules.The FF evaluation and genetic evolution take part in an iterative procedure, which ends when a maximum number of generations are reached.

Assuming an initial random population produced and evaluated, genetic evaluation takes place by means of three basic genetic operators:

a) Parent selection

b) Crossover

c) Mutation

a) Parent selection is a simple procedure where by two chromosomes are selected from the parent population based on their fitness value. Solutions with high fitness values have a high probability of controlling new offspring to the next generation. The selection rule used in this paper is a simple roulette-wheel selection.

b) Crossover is an extremely important operator for the GA. It is responsible for the structure recombination (information exchange between mating chromosomes) and the convergence speed of the GA and is usually applied with high probability $(0.6-0.9)$. The chromosomes of the two parents selected are combined to form new chromosomes that inherit segments or information stored in parent chromosomes. Until now, many crossover schemes, such as single point, multipoint, or uniform c) Mutation is the operator responsible for the injection of new information. With a small probability, random bits of the offspring chromosomes flip from 0 to 1 and vice versa and give new characteristics that do not exist in the parent population. In this paper, the mutation operator is applied with a relatively small probability $(0.001-0.005)$ to every bit of the chromosome. Operations of selection, crossover and mutation are repeated until the number of desired off springs is created. The objective function is then calculated for every off springs and the best individuals among the entire pool, comprising parents and their off springs, are kept to constitute the new generation. The final result of the GA optimization is the best individual of the last iteration.

The termination criteria for GA will be any one of the following two conditions:

i). The maximum number of generations is achieved.

ii). When the genotype of the population of individuals converges, the convergences of the genotype structure occur when all bit positions in all string are identical. In this case, crossover will have no further effect.

The steps to follow for GA is as shown in the flow chart as in fig. 1 .

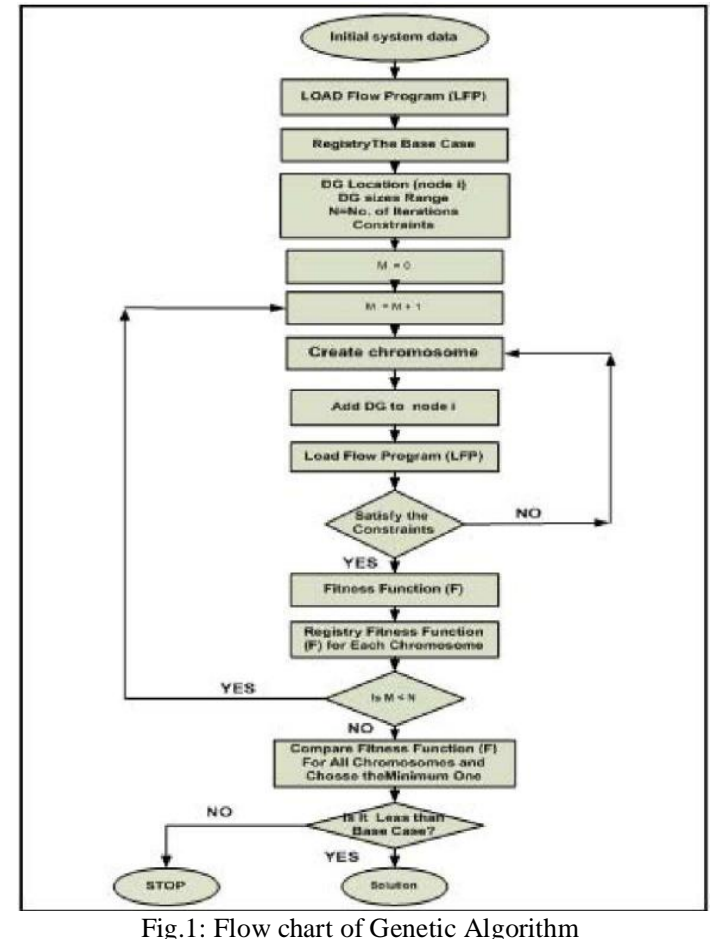

\section{PROBLEM FORMULATION}

In this paper, the number, location and size of DG units are decided in such way that minimum system power loss and desired voltage profile is obtained. So it is needed to define system power loss as a function of DG size and system bus voltages. 


\section{A. Objective Function}

A precise evaluation for the Objective Function has been selected. The main goal of the proposed algorithm is to determine the best locations and size for new Distributed Generation resources by minimizing different function, related to project aims. Two main goals are taken into considerations to determine the Objective Formula that is used in point of start: Power Losses reduction and voltage profile improvement. The Fitness Function is determined as following:

$$
\mathrm{F}=\mathrm{WP} * \mathrm{PL}+\mathrm{Wq} * \mathrm{PL}+\mathrm{Wq} * \mathrm{CVD}
$$

Where: PL: Active Power Loss

QL: Reactive Power Loss

CVD: Cumulative Voltage Deviation

F: Fitness Function.

The active and reactive power losses are obtained from load flow program. The cumulative voltage deviation norm is defined as "the normalized sum of the deviations of the obtained value from the desired value at every node on the feeder. The desired value being $1.0 \mathrm{p}$.u and the obtained value being the value obtained from the threephase distribution power flow. In this work the CVD is determined the same way as following:

$\mathrm{CVD}=\sum_{I=N}\left|1-V_{I}\right|$

B. Constraints The main constraints in the optimization process in the proposed methodology are:

1. Active and reactive power losses constraints

2. Voltage Constraints

\section{Active and reactive power losses constraint:}

The losses after installing DG in power grid should be less than or equal losses before installing DGs.

$\mathrm{PL}$ with $\mathrm{DG} \leq \mathrm{PL}$ without DG

$\mathrm{QL}$ with $\mathrm{DG} \leq \mathrm{QL}$ without $\mathrm{DG}$

Voltage constraint: To ensure the voltage of any bus should be within predefined limits the following constraint is considered:

$\mathrm{V}$ bus $\min \leq \mathrm{V}$ bus $\leq \mathrm{V}$ bus $\max$

$\mathrm{V}$ bus $=$ bus voltage

$\mathrm{V}$ bus $\max =$ maximum bus voltage

$\mathrm{V}$ bus $\min =$ minimum bus voltage

C. Wei bull probability density function: The Weibull probability density function (PDF) used in this study to characterize diameter distribution is of the following form:

$$
f(x)=\left[\frac{\beta}{\theta}\left(\frac{x-\delta}{\theta}\right)^{\beta-1}\right]\left\{e^{-\left[\left(\frac{x-\delta}{\theta}\right)\right]^{\beta}}\right\}, x \geq \delta
$$

\section{Net Present Value:}

The Net Present Value (NPV) approach uses the time value of money to convert future cash flow into a present value at a certain discount rate. Therefore, this approach is also called Discounted Cash Flow (DCF). Due to the time value of money, a hundred dollars today are more valuable than a hundred dollars in the future, see Figure 2.5. Mathematically, the present value of future cash flow is defined by the following formula :

$$
P V=F V /(1+D R) n
$$

where PV and FV are the Present and the Future Values, respectively; DR is discount rate, and $\mathrm{N}$ is number of years in the future.

\section{RESULTS AND DISCUSSION}

The proposed model has been implemented and tested on IEEE 37-bus test system. Fitness value is calculated at all buses. The bus with high fitness value was used as optimal location. DG size was tested in the range of 0.5 to $5 \mathrm{MW}$ with the step size of 0.5. Optimal size was obtained as $2.5 \mathrm{MW}$. The various fitness values have been shown in the table and figures below.

\begin{tabular}{|l|l|}
\hline BUSNUMBER & FITNESS VALUES \\
\hline 3 & 4.3088 \\
\hline 4 & 1.1695 \\
\hline 5 & 1.9562 \\
\hline
\end{tabular}

Table1: fitness values of $\mathrm{dg}$ allocated buses

The table shows only the fitness values which are exceeding the constrains where we place DG and remaining all buses the fitness values are less than one. The Fig 2 shows the variation of fitness values for different buses using GA

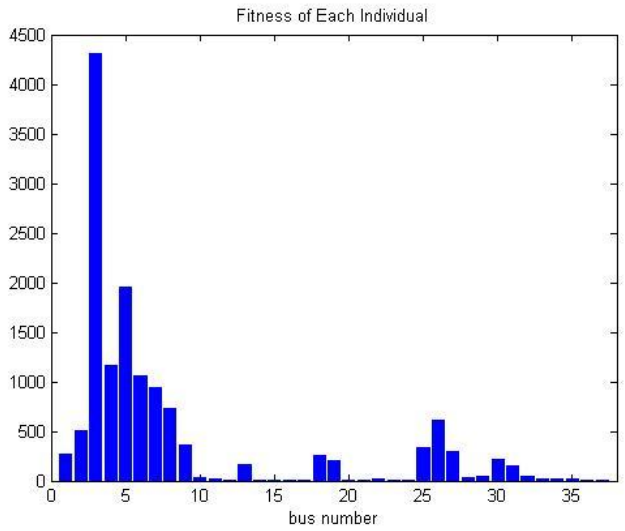

Fig 2: Fitness values for IEEE37 bus system The Optimal placement of DG is find out with this fitness values .By observing above fitness values the values of bus 3 ,bus 4 and bus 5 exceeds the value 1 so necessary placement of DG at these buses to improve the voltage profile and reduces active power losses. The respective fitness plot is shown in figure 4.1 as below using Genetic Algorithm (GA). The power losses in the IEEE 37 bus system with and without DG is shown in table 4.2 below

\begin{tabular}{|l|l|l|l|}
\hline $\begin{array}{l}\text { Bus } \\
\text { system }\end{array}$ & $\begin{array}{l}\text { Real } \\
\text { power } \\
\text { loss } \\
(\mathrm{KW})\end{array}$ & $\begin{array}{l}\text { Reactive } \\
\text { power loss } \\
(\mathrm{KW})\end{array}$ & $\begin{array}{l}\text { Real power } \\
\text { loss (using } \\
\mathrm{DG}(\mathrm{KW})\end{array}$ \\
\hline 37 & 31.509 & 28.01 & 27.73650 \\
\hline
\end{tabular}

Table 2 System Power Losses In Absence \& presence Of Dg Units 
By Observing the active power losses can be decreased with the installation of wind based DG at optimal bus location. If wrong placement of DG causes increase in real power loss and voltage profile and causes adverse effects.

The figure 3 shows the variation of NPV with respect to the Risk Factor (RF) [hrs/year] . NPV will decreases as the Risk Factor increases but when compare to The Net Present Worth(NPV) value with the placement of DG does not decrease abruptly as the risk factor increases(say for 10 years) shown in below

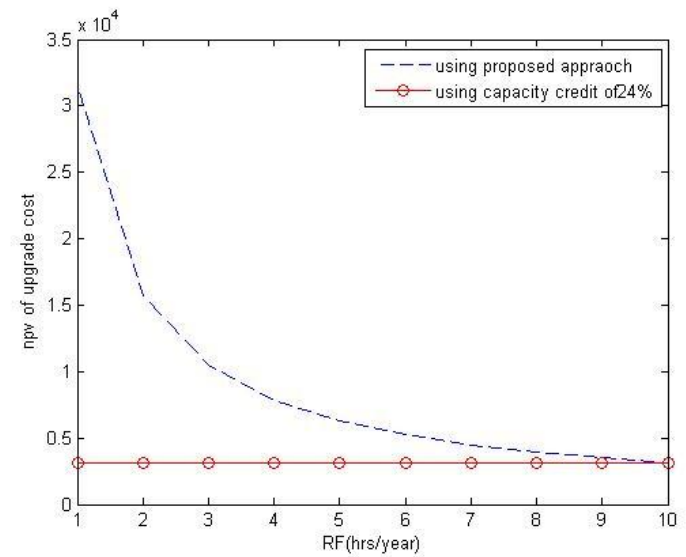

Fig 3 NPV upgrade cost with and without wind based DG

The Fig 4 shows he variation of wind speed for different months in a year for that we are selecting weibull probability density function for different values of $\beta$ the plots shows the wind speed variations with respect to $\beta$ values.

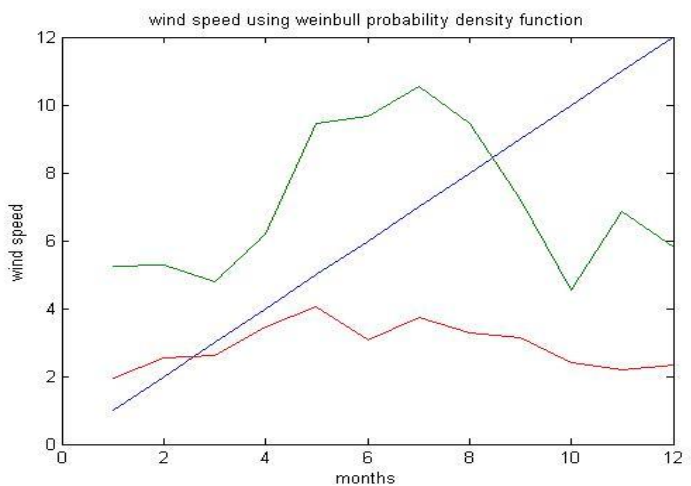

Fig :4 The variation of wind speed using wei bull probability density function

\section{CONCLUSION}

This paper presents a methodology for solving the best location and sizing of DG problem in unbalanced radial distribution. The effectiveness of the algorithm has been demonstrated and tested. The sizing of DG has been obtained with an objective function of reducing power losses. The proposed methodology was successfully applied to IEEE 37 node test feeders. The obtained solution has been successful in reducing total active power losses in 37 node RDS. Thus the proposed method has been observed as efficient for solving DG placement and sizing in radial distribution systems.

\section{REFERENCES}

[1] N.Mithulananthan,than OO and LE van phu, "Distributed Generator Placement In Power Distribution System Using Genetic Algorithm To Reduce Losses", Thammasat Int.J.Sc. Tech., vol. 9, no. 3, JulySeptember 2004.

[2] T. Ackermann, G. Andersson, and L.Söder, "Distributed Generation: A Definition Proceedings Of The First International Symposium On Distributed Generation And Market Aspects, June 11-13, 2001, Royal Institute Of Technology, Sweden

[3] A. Piccolo and P. Siano, "Evaluating the impact of network investmentdeferral on distributed generation expansion," IEEE Trans. Power Syst., vol. 24, no. 3, pp. 1559-1567, Aug. 2009.

[4] S. H. Jangamshetti and V. G. Rau, "Site matching of wind turbine generators: A case study," IEEE Trans. Energy Convers., vol. 14, no. 4,pp. 1537-1543, Dec. 1999.

[5] W. El-Khattam, and M.M.A. Salama, "Distributed Generation Technologies, Definitions And Benefits", Electric Power Systems Research. 71, pp.119-128, 2004.

[6] C. Chiang, "Genetic-based algorithm for power economic load dispatch,"IET Gen., Transm., Distrib., vol. 1, no. 2, pp. 261-269, Mar. 2007.

[7] Yiming Mao, and Karen N. Miu ,"Switch Placement to Improve System Reliability for Radial Distribution Systems with Distributed Generation" , IEEE TRANSACTIONS ON POWER SYSTEMS, VOL. 18, NO. 4, NOVEMBER 2003.

[8] A. L. Devi, B. Subramanyam, "Sizing of DG Unit Operated at Optimal Power Factor to Reduce Losses in Radial Distribution. A Case Study," Journal of Theoretical and Applied Information Technology (JATIT), $2005-2008$.

[9] S. Ghosh , S.P. Ghoshal and S. Ghosh. Optimal sizing and placement of DG in network system.International Journal of Electrical Power and Energy Systems. 2010, 32 (8): 849-856.

[10] M. Padma Lalitha, V.C. Veera Reddy, N. Usha. 2009. DG placement using Fuzzy for maximum loss reduction in radial distribution system. International journal of computers applications in engineering, technology and sciences.

[11] M. Gandomkar,M. Vakilian,M. Ehsan, " A combination of genetic algorithm and simulated annealing for optimal DG allocation in distribution networks", CCECE/CCGEI,Saskatoon, May 2005 IEEE, PP.645-648.

[12] D. Singh, D. Singh and K.S. Verma. Multi-objective optimization for DG planning with load models.IEEE Transactions on Power Systems. 2009, 24 (1): 427-436.

[13] R.M. Kamel and B. Karmanshahi.Optimal size and location of DGs for minimizing power losses in a primary distribution network.Transaction on Computer Science and Electrical and Electronics Engineering. 2009, 16 (2): 137-144. 